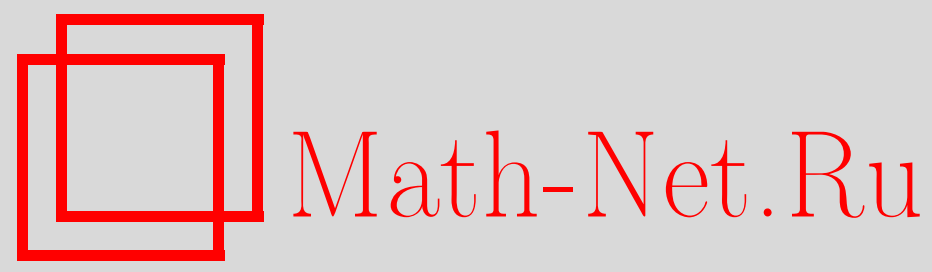

А. Б. Секерин, Теорема о носителе для комплексного преобразования Радона обобщенных функций, Матем. заметки, 2003, том 74, выпуск 5, 719-727

DOI: https://doi.org/10.4213/mzm304

Использование Общероссийского математического портала Math-Net.Ru подразумевает, что вы прочитали и согласны с пользовательским соглашением http://www . mathnet.ru/rus/agreement

Параметры загрузки:

IP: 54.84 .234 .179

26 апреля 2023 г., 16:10:48

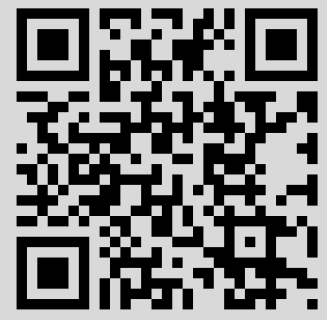




\section{ТЕОРЕМА О НОСИТЕЛЕ ДЛЯ КОМПЛЕКСНОГО ПРЕОБРАЗОВАНИЯ РАДОНА ОБОБЩЕННЫХ ФУНКЦИЙ}

\section{А. Б. Секерин}

Рассматриваются свойства комплексного преобразования Радона распределений с компактным носителем. Для таких распределений доказывается теорема о носителе, позволяющая описать носитель распределения по носителю его преобразования Радона.

Библиографияя: 9 названий.

Классическое действительное (комплексное) преобразование Радона сопоставляет достаточно быстро убывающей непрерывной функции набор интегралов от нее по всем действительньм (комплексным) гиперплоскостям [1]. Будем говорить, что для компакта $K \subset \mathbb{R}^{n}\left(\mathbb{C}^{n}\right)$ верна теорема о носителе для преобразования Радона, если любая финитная непрерьвная функция $\varphi$ равна нулю вне $K$ при условии, что равны нулю интегралы от этой функции по всем действительным (комплексным) гиперплоскостям, не пересекающим $K$. В случае действительного преобразования Радона теорема о носителе доказана для любого выпуклого компакта $K$ [2], [3].

Для комплексного преобразования Радона теоремы о носителе имеет смысл рассматривать для компактов, линейно выпуклых по Мартино. Напомним, что линейно выпуклым по Мартино называется компакт $K \subset \mathbb{C}^{n}$ такой, что для каждой точки $z \notin K$ существует комплексная гиперплоскость, содержащая $z$ и не пересекающая $K$. В работе автора [4] выделен класс компактов, линейно вьпуклых по Мартино, для которых верна теорема о носителе. Целью данной работы является доказательство теоремы о носителе для комплексного преобразования Радона распределений с компактным носителем.

Через $B^{n}(z, R)$ и $\bar{B}^{n}(z, R)$ будем соответственно обозначать открытый и замкнутьй шар радиуса $R$ в $\mathbb{C}^{n}$ с центром в точке $z$. Единичная сфера в $\mathbb{C}^{n}$ обозначается через $S^{2 n-1}, d \sigma$ - элемент площади сферы, $d \omega_{2 n}-$ стандартная мера Лебега в $\mathbb{C}^{n}$.

Следуя Л. Шварцу, через $\mathscr{S}\left(\mathbb{C}^{n}\right)$ и $\mathscr{D}\left(\mathbb{C}^{n}\right)$ будем обозначать пространства функций класса $C^{\infty}\left(\mathbb{C}^{n}\right)$, быстро убьвающих со всеми производными и, соответственно, имеющих компактный носитель; $\mathscr{E}\left(\mathbb{C}^{n}\right)-$ пространство $C^{\infty}\left(\mathbb{C}^{n}\right)$, наделенное топологией равномерной сходимости функций и их производных на компактах. Классическое комплексное преобразование Радона функции $\varphi \in \mathscr{S}\left(\mathbb{C}^{n}\right)$ задается равенством

$$
\hat{\varphi}(s, \xi)=\frac{1}{|\xi|^{2}} \int_{\left\langle z, \frac{\xi}{|\xi|}\right\rangle=\frac{s}{|\xi|}} \varphi(z) d \lambda(z), \quad(s, \xi) \in \mathbb{C} \times\left(\mathbb{C}^{n} \backslash 0\right),
$$

Работа выполнена по гранту Минобразования РФ Е00-1.0-105. 
где $d \lambda$ - элемент площади на гиперплоскости $z:\langle z, \xi /|\xi|\rangle=s /|\xi|$.

Пусть $C^{\infty}\left(\mathbb{C} \times S^{2 n-1}\right)$ - пространство функций $h(s, w)$ таких, что частные производные любого порядка

$$
\frac{\partial^{k_{1}+k_{2}}}{\partial s^{k_{1}} \partial \bar{s}^{k_{2}}} h(s, w)
$$

существуют и непрерывны по совокупности переменных. Через $\mathscr{D}\left(\mathbb{C} \times S^{2 n-1}\right)$ будем обозначать пространство функций из $C^{\infty}\left(\mathbb{C} \times S^{2 n-1}\right)$, носители которых - компакты в $\mathbb{C} \times S^{2 n-1}$. Пусть далее $\mathscr{R} \mathscr{D}$ - подпространство в $\mathscr{D}\left(\mathbb{C} \times S^{2 n-1}\right)$, образованное преобразованиями Радона функций из $\mathscr{D}\left(\mathbb{C}^{n}\right)$.

Пространство $\mathscr{R} \mathscr{D}$ допускает внутреннее описание. Функция $\varphi(s, w)$ принадлежит пространству $\mathscr{R} \mathscr{D}$ тогда и только тогда, когда вьполнены следующие условия:

а) функция $\varphi(s, w)$ является сужением на $\mathbb{C} \times S^{2 n-1}$ функции $\varphi(s, \xi) \in C^{\infty}(\mathbb{C}$ $\left.\times\left(\mathbb{C}^{n} \backslash 0\right)\right)$, удовлетворяющей для любого комплексного $\alpha \neq 0$ условию однородности $\varphi(\alpha s, \alpha \xi)=|\alpha|^{-2} \varphi(s, \xi)$;

б) функция $\varphi(s, \xi)$ удовлетворяет условиям аналога теоремы Пэли-Винера для преобразования Радона [1, с. 170];

в) для некоторого $R>0$ функция $\varphi(s, w)$ обращается в нуль на множестве $\{|s|>R\}$ $\times S^{2 n-1}$.

Условия а) и б) представляют собой необходимое и достаточное условие того, что функция $\varphi(s, w)$ является преобразованием Радона некоторой функции $f(z)$ из $\mathscr{S}\left(\mathbb{C}^{n}\right)[1$, c. 170]. Из условия в) следует тогда, что равны нулю интегралы от функции $f(z)$ по всем комплексным гиперплоскостям, не пересекающим шар радиуса $R$ с центром в нуле. Отсюда очевидно вытекает, что равны нулю интегралы от функции $f(z)$ по всем действительньп гиперплоскостям, не пересекающим этот шар. Тогда по теореме Хелгасона о носителе для действительного преобразования Радона $[2$, с. 39] $\operatorname{supp}(f) \subset\{|z| \leqslant R\}$.

Зададим на $C^{\infty}\left(\mathbb{C} \times S^{2 n-1}\right)$ стандартную топологию, определяемую системой полунорм

$$
q_{k}(f)=\max _{k_{1}+k_{2} \leqslant k} \max _{|s| \leqslant k} \max _{w \in S^{2 n-1}}\left|\frac{\partial^{k_{1}+k_{2}} f(s, w)}{\partial s^{k_{1}} \partial \bar{s}^{k_{2}}}\right| .
$$

Пространство $C^{\infty}\left(\mathbb{C} \times S^{2 n-1}\right)$ с такой топологией будем обозначать через $\mathscr{E}\left(\mathbb{C} \times S^{2 n-1}\right)$. Пусть

$$
R^{*}: \mathscr{E}\left(\mathbb{C} \times S^{2 n-1}\right) \rightarrow \mathscr{E}\left(\mathbb{C}^{n}\right)
$$

оператор дуального преобразования Радона:

$$
\left[R^{*}(f)\right](z)=\int_{S^{2 n-1}} f(\langle z, w\rangle, w) d \sigma(w)
$$

Нетрудно видеть, что оператор $R^{*}$ непрерьвен. Кроме того, справедлива следующая формула $[5$, с. 10$]$ :

$$
\int_{\mathbb{C}^{n}}\left[R^{*}(f)\right](z) \varphi(z) d \omega_{2 n}(z)=\int_{\mathbb{C}} \int_{S^{2 n-1}} f(s, w) \hat{\varphi}(s, w) d \sigma(w) d \omega_{2}(s),
$$

где $\varphi(z)$ - любая функция из $\mathscr{D}\left(\mathbb{C}^{n}\right), \hat{\varphi}(s, w)$ - преобразование Радона функции $\varphi$. 
Заметим, что для любого $\theta \in[0,2 \pi]$ значения оператора $R^{*}$ на функциях $f(s, w)$ $\in \mathscr{E}\left(\mathbb{C} \times S^{2 n-1}\right)$ и $f\left(s e^{i \theta}, w e^{i \theta}\right)$ совпадают. Поэтому, полагая для функции $f(s, w)$ $\in \mathscr{E}\left(\mathbb{C} \times S^{2 n-1}\right)$

$$
\tilde{f}(s, w)=\frac{1}{2 \pi} \int_{0}^{2 \pi} f\left(s e^{i \theta}, w e^{i \theta}\right) d \theta
$$

получаем

$$
R^{*}(\tilde{f})=R^{*}(f)
$$

Таким образом, оператор $R^{*}$ фактически задан на функциях из $\mathscr{E}\left(\mathbb{C} \times S^{2 n-1}\right)$, удовлетворяющих условию

$$
h\left(e^{i \theta} s, e^{i \theta} w\right) \equiv h(s, w), \quad \theta \in[0,2 \pi] .
$$

Пусть $M-$ пространство функций вида

$$
\psi(s, w)=\frac{\partial^{2 n-2}}{\partial s^{n-1} \partial \bar{s}^{n-1}} \hat{\varphi}(s, w), \quad \hat{\varphi}(s, w) \in \mathscr{R} \mathscr{D} .
$$

Функция $\varphi(z) \in \mathscr{D}\left(\mathbb{C}^{n}\right)$ выражается через свое преобразование Радона $\hat{\varphi}(s, w)$ по формуле [1, с. 165]

$$
\varphi=(-1)^{n-1} c_{n} R^{*}\left(\frac{\partial^{2 n-2}}{\partial s^{n-1} \partial \bar{s}^{n-1}} \hat{\varphi}\right)
$$

где число $c_{n}>0$ зависит только от размерности $n$. Поэтому, если $\psi \in M$, то функция $\varphi(z)$, преобразование Радона $\hat{\varphi}(s, w)$ которой связано с функцией $\psi(s, w)$ равенством (5), определяется единственным образом:

$$
\varphi(z)=(-1)^{n-1} c_{n} R^{*}(\psi)
$$

Имеется ряд различных подходов к понятию преобразования Радона обобщенных функций [1]-[3], [6]. Мы будем использовать подход, предложенный в монографии И. М. Гельфанда и др. [1]:

ОПРЕДЕЛЕНИЕ [1]. Преобразованием Радона распределения $F \in \mathscr{D}^{\prime}$ назьвается функционал $R F$ на пространстве $M$, определяемьй равенством

$$
\langle R F, \psi\rangle=\left\langle F, R^{*}(\psi)\right\rangle \text {. }
$$

В зависимости от пространства основных функций $\left(\mathscr{D}\left(\mathbb{C} \times S^{2 n-1}\right), \mathscr{E}\left(\mathbb{C} \times S^{2 n-1}\right)\right.$ и др.) в дальнейшем под преобразованием Радона функиионала $F$ будем понимать продолжение функционала $R F$ с пространства $M$ на соответствующее пространство. Данное продолжение, вообще говоря, неоднозначно. Для основных функций $\varphi \in \mathscr{S}\left(\mathbb{C}^{n}\right)$ обобщенное преобразование Радона совпадает с обычньм $[1$, с. 171] (в этом случае функционал $R \varphi$ формулой (7) задается на всем пространстве $\left.\mathscr{S}\left(\mathbb{C} \times S^{2 n-1}\right)\right)$.

Для любой функции $f \in \mathscr{E}\left(\mathbb{C} \times S^{2 n-1}\right)$ имеем $R^{*}(f) \in \mathscr{E}\left(\mathbb{C}^{n}\right)$. Если $F \in \mathscr{E}^{\prime}\left(\mathbb{C}^{n}\right)$ - распределение с компактным носителем, то $R F$ формулой $(7)$ задается на всем пространстве $\mathscr{E}\left(\mathbb{C} \times S^{2 n-1}\right)$. Таким образом, в случае $F \in \mathscr{E}^{\prime}\left(\mathbb{C}^{n}\right)$ функционал $R F$ непосредственно продолжается с пространства $M$ на $\mathscr{E}\left(\mathbb{C} \times S^{2 n-1}\right)$. При этом в силу непрерывности $R^{*}$ данное продолжение непрерьвно в заданной вьше топологии $\mathscr{E}\left(\mathbb{C} \times S^{2 n-1}\right)$. Именно это продолжение будет в дальнейшем подразумеваться под преобразованием Радона 
распределения с компактным носителем. Далее, в силу непрерьвности $R F$ для некоторых $C>0$ и $k$ имеем $|R(F)(f)| \leqslant C q_{k}(f)$, где полунормы $q_{k}(f)$ определены соотношением (2). Тогда, если носитель функции $f(s, w) \in \mathscr{E}\left(\mathbb{C} \times S^{2 n-1}\right)$ не пересекается с множеством $\{|s| \leqslant k\} \times S^{2 n-1}$, то $R F(f)=0$, т.е. носитель функционала $R F$ содержится в $\{|s| \leqslant k\} \times S^{2 n-1}$. Таким образом, преобразование Радона распределения с компактным носителем также имеет компактньй носитель.

В достаточно общей ситуации можно указать необходимое и достаточное условие компактности носителя исходного функционала при условии компактности носителя его преобразования Радона. Рассмотрим любую функцию $h(s, w) \in \mathscr{D}\left(\mathbb{C} \times S^{2 n-1}\right)$. Пусть

$$
u(s, w)=\frac{\partial^{2 n-2}}{\partial s^{n-1} \partial \bar{s}^{n-1}} h(s, w), \quad F(z)=(-1)^{n-1} c_{n}\left[R^{*}(u)\right](z) .
$$

$\Phi$ ункция $F(z)$ является бесконечно дифференцируемой функцией в $\mathbb{C}^{n}$. Поэтому мы можем рассматривать ее как обобщенную функцию и задать еепреобразование Радона $R F$ в соответствии с приведенным выше определением. В этих обозначениях справедливо следующее утверждение.

ПРЕДЛОЖЕНИЕ 1. Преобразование Радона $R F$ функиии $F$ совпадает $c h(s, w)$. Функиия $F(z)$ имеет компактный носитель тогда и только тогда, когда функиия $h(s, w)$ принадлехит пространству $\mathscr{R} \mathscr{D}$, т.е. является преобразованием Радона некоторой функиии из $\mathscr{D}\left(\mathbb{C}^{n}\right)$.

ДокАЗАТЕЛЬСтво. Пусть $M$ - введенное выше пространство и $\psi(s, w) \in M$. По определению $M$ имеем

$$
\psi(s, w)=\frac{\partial^{2 n-2}}{\partial s^{n-1} \partial \bar{s}^{n-1}} \hat{\varphi}(s, w),
$$

где $\hat{\varphi}(s, w)$ - преобразование Радона некоторой функции $\varphi \in \mathscr{D}\left(\mathbb{C}^{n}\right)$. По формуле обращения (6) $\varphi(z)=c_{n}(-1)^{n-1}\left[R^{*}(\psi)\right](z)$. Тогда по построению функции $F(z)$ верно

$$
\left\langle F, R^{*}(\psi)\right\rangle=\left\langle(-1)^{n-1} c_{n}\left[R^{*}(u)\right](z),\left[R^{*}(\psi)\right](z)\right\rangle=\left\langle\left[R^{*}(u)\right](z), \varphi(z)\right\rangle .
$$

Используя (3), получаем

$$
\left\langle F, R^{*}(\psi)\right\rangle=\int_{\mathbb{C}} \int_{S^{2 n-1}} u(s, w) \hat{\varphi}(s, w) d \sigma(w) d \omega_{2}(s) .
$$

Поэтому из (8) следует

$$
\left\langle F, R^{*}(\psi)\right\rangle=\int_{\mathbb{C}} \int_{S^{2 n-1}} h(s, w) \frac{\partial^{2 n-2}}{\partial s^{n-1} \partial \bar{s}^{n-1}} \hat{\varphi}(s, w) d \sigma(w) d \omega_{2}(s) .
$$

Таким образом, для любой функции $\psi(s, w) \in M$ имеем

$$
\left\langle F, R^{*}(\psi)\right\rangle=\int_{\mathbb{C}} \int_{S^{2 n-1}} h(s, w) \psi(s, w) d \sigma(w) d \omega_{2}(s) .
$$

Это означает, что в соответствии с (7) преобразование Радона $R F$ функционала $F$ задается функцией $h(s, w)$ и, в частности, имеет компактный носитель. 
Докажем второе утверждение. Пусть $h(s, w) \in \mathscr{R} \mathscr{D}$, т.е. $h(s, w)$ - преобразование Радона некоторой функции $h(z) \in \mathscr{D}\left(\mathbb{C}^{n}\right)$. Тогда по построению функции $F(z)$ и в силу формулы обрашения (6) имеем $F(z) \equiv h(z)$, т.е. $F(z)$ - функция с компактным носителем. Обратно, предположим, что функция $F(z)$ имеет компактный носитель. Тогда $F \in \mathscr{D}\left(\mathbb{C}^{n}\right)$. С одной стороны, для любой функции $\varphi(z) \in \mathscr{D}\left(\mathbb{C}^{n}\right)$ имеем в силу (3)

$$
\begin{aligned}
\langle F, \varphi\rangle & =\left\langle(-1)^{n-1} c_{n}\left[R^{*}(u)\right](z), \varphi\right\rangle \\
& =(-1)^{n-1} c_{n} \int_{\mathbb{C}} \int_{S^{2 n-1}} u(s, w) \hat{\varphi}(s, w) d \sigma(w) d \omega_{2}(s) .
\end{aligned}
$$

$\mathrm{C}$ другой стороны, так как $F \in \mathscr{D}\left(\mathbb{C}^{n}\right)$, по формуле Планшереля для преобразования Радона $[1$, с. 168$]$ имеем

$$
\langle F, \varphi\rangle=(-1)^{n-1} c_{n} \int_{\mathbb{C}} \int_{S^{2 n-1}} \frac{\partial^{2 n-2}}{\partial s^{n-1} \partial \bar{s}^{n-1}} \widehat{F}(s, w) \hat{\varphi}(s, w) d \sigma(w) d \omega_{2}(s),
$$

где $\widehat{F}(s, w)$ - преобразование Радона функции $F(z)$. Тогда из (9) следует

$$
\int_{\mathbb{C}} \int_{S^{2 n-1}}\left(\frac{\partial^{2 n-2}}{\partial s^{n-1} \partial \bar{s}^{n-1}} \widehat{F}(s, w)-u(s, w)\right) \hat{\varphi}(s, w) d \sigma(w) d \omega_{2}(s)=0
$$

для любой функции $\varphi \in \mathscr{D}\left(\mathbb{C}^{n}\right)$. Таким образом, функция

$$
v(s, w)=\frac{\partial^{2 n-2} \widehat{F}(s, w)}{\partial s^{n-1} \partial \bar{s}^{n-1}}-u(s, w)=\frac{\partial^{2 n-2}(\widehat{F}(s, w)-h(s, w))}{\partial s^{n-1} \partial \bar{s}^{n-1}}
$$

ортогональна $\mathscr{R} \mathscr{D}$. Тогда $[7$, c. 49$]$ для любой сферической гармоники $Y_{m}(w)$ степени $m$ функция

$$
a_{m}(s)=\int_{S^{2 n-1}} v(s, w) Y_{m}(w) d \sigma(w)
$$

представляет собой многочлен, степени строго меньшей $m$. Так как функция $v(s, w)$ имеет компактный носитель, то $a_{m}(s)=0$ для $|s|>R$, но тогда $a_{m}(s) \equiv 0$. Таким образом, при любом фиксированном $s$ функция $v(s, w)$ ортогональна на сфере всем сферическим гармоникам. Следовательно, $v(s, w) \equiv 0$. Поэтому

$$
\frac{\partial^{2 n-2}(\widehat{F}(s, w)-h(s, w))}{\partial s^{n-1} \partial \bar{s}^{n-1}} \equiv 0 .
$$

Так как функция $\widehat{F}(s, w)-h(s, w)$ финитнапо $s$, отсюда следует, что $\widehat{F}(s, w)-h(s, w) \equiv 0$, т.е. $h(s, w) \in \mathscr{R} \mathscr{D}$. Предложение доказано.

Из приведенного выше описания пространства $\mathscr{R} \mathscr{D}$ и аналога теоремы Пэли-Винера для преобразования Радона следует, что $\mathscr{R} \mathscr{D}$ является собственным подпространством в пространстве функций из $\mathscr{D}\left(\mathbb{C} \times S^{2 n-1}\right)$, удовлетворяющих соотношению (4). Поэтому, если в обозначениях предложения 1 функция $h(s, w)$ не принадлежит пространству $\mathscr{R} \mathscr{D}$, то из предложения 1 вытекает, что носитель функционала, задаваемого функцией $F(z)$, не компактен, несмотря на то что его преобразование Радона имеет компактный носитель.

Рассмотрим задачу восстановления носителя функционала $F \in \mathscr{E}^{\prime}\left(\mathbb{C}^{n}\right)$ по носителю его преобразования Радона. Для множества $A \subset \mathbb{C}^{n}$ через $\widehat{A}$ будем обозначать множество таких $(s, w) \in \mathbb{C} \times S^{2 n-1}$, что гиперплоскость $z:\langle z, w\rangle=s$ пересекает множество $A$. Если $A$ открытое множество или компакт в $\mathbb{C}^{n}$, то множество $\widehat{A}$ соответственно открыто или компактно в $\mathbb{C} \times S^{2 n-1}[8]$. 
ТеОрема 1. Пусть $K \subset \mathbb{C}^{n}$ - компакт, линейно выпуклый по Мартино. Предположим, что для каждой точки $z \notin K$ существует комплексная гиперплоскость $P=\left\{\lambda:\left\langle\lambda, \xi_{0}\right\rangle=s_{0}\right\}$, содержащая точку z и удовлетворяющая следующим условиям:

a) $P \cap K=\varnothing$;

б) множсество $\mathbb{C} \backslash K_{\xi_{0}}$ связно, где $K_{\xi_{0}}=\left\{\left\langle\lambda, \xi_{0}\right\rangle\right\}_{\lambda \in K}-$ проекиия компакта $K$ на $\xi_{0}$.

Пусть $F \in \mathscr{E}^{\prime}\left(\mathbb{C}^{n}\right)$ - распределение с компактным носителем такое, что носитель его преобразования Радона $R F$ содерэится в $\widehat{K}$. Тогда $\operatorname{supp} F \subset K$.

Отметим, что априорное требование компактности носителя распределения $F$ (принадлежность $F$ пространству $\left.\mathscr{E}^{\prime}\left(\mathbb{C}^{n}\right)\right)$ существенно в силу предложения 1 и сделанного выше замечания. Следует также отметить, что теорема 1 не предполагает связности компакта $K$.

Для случая, когда распределение $F$ задается непрерьвной функцией, теорема 1 доказана в работе [4]. Кроме того, в этой работе приведен пример связного компакта, линейно вьпуклого по Мартино, для которого теорема о носителе неверна. Этот пример показывает, что условия, налагаемые теоремой 1 на компакт $K$, существенны. В действительном случае под линейно вьпуклым компактом можно понимать такой компакт $K$, что через каждую точку, лежащую вне $K$, проходит действительная гиперплоскость, не пересекающая $K$. Для связного компакта это свойство эквивалентно обычной выпуклости. Поскольку для выпукльх компактов теорема о носителе действительного преобразования Радона верна, то упомянутьй вьше пример показьвает также, что между свойствами носителей действительного и комплексного преобразований Радона нет полной аналогии.

В работе [8] доказана следующая теорема, которая будет лежать в основе доказательства теоремы 1:

ТЕОрема 2. Пусть $\varphi(z)$ - непрерывная в $\mathbb{C}^{n}$ функиия с компактным носителем, $\hat{\varphi}(s, w)$ - комплексное преобразование Радона $\varphi$. Пусть точка $\left(w_{0}, s_{0}\right)$ не принадлехит носителю $\hat{\varphi}$. Пусть далее существует открытое, связное, неограниченное множество $D \subset \mathbb{C}$ такое, что $s_{0} \in D$ и пересечение $\left(D \times\left\{w_{0}\right\}\right) \cap \operatorname{supp} \hat{\varphi}$ пусто. Тогда функция $\varphi$ равна нулю на гиперплоскости $\left\{z:\left\langle z, w_{0}\right\rangle=s_{0}\right\}$.

ДОКАЗАТЕЛЬСТВО ТЕОРЕМЫ 1. В следующих ниже рассуждениях будут использоваться свойства свертки распределений с гладкими функциями $[9$, с. 112,113$]$. Пусть $\alpha_{\varepsilon}$, $0<\varepsilon \leqslant 1,-$ стандартное семейство функций из $\mathscr{D}\left(\mathbb{C}^{n}\right)$, аппроксимирующих $\delta$-функцию, т.е. выполняются следующие условия: $0 \leqslant \alpha_{\varepsilon}, \operatorname{supp} \alpha_{\varepsilon} \subset\{|z| \leqslant \varepsilon\}$,

$$
\int_{\mathbb{C}^{n}} \alpha_{\varepsilon}(z) d \omega_{2 n}(z)=1
$$

Пусть $F \in \mathscr{E}^{\prime}\left(\mathbb{C}^{n}\right)$ и $\operatorname{supp} R F \subset \widehat{K}$. Рассмотрим обобщенную функцию $F_{\varepsilon}=F * \alpha_{\varepsilon}$. $\Phi$ ункционал $F_{\varepsilon}$ задается гладкой функцией с компактным носителем, которую будем обозначать через $F_{\varepsilon}(z)$. Преобразование Радона $R F_{\varepsilon}$ функционала $F_{\varepsilon}$ - регулярное распределение, задаваемое функцией $\widehat{F}_{\varepsilon}(s, w)$. Для описания носителя функции $\widehat{F}_{\varepsilon}(s, w)$ понадобится следующая 
Лемма 1. Для любой функиии $\psi \in \mathscr{E}\left(\mathbb{C} \times S^{2 n-1}\right)$ верно

$$
\alpha_{\varepsilon} * R^{*}[\psi]=R^{*}\left[\left(\hat{\alpha}_{\varepsilon} *_{s} \psi\right)(s, w)\right],
$$

где $R^{*}$ - оператор дуального преобразования Радона, $\hat{\alpha}_{\varepsilon}(s, w)$ - преобразование Радона функиии $\alpha_{\varepsilon}(z)$, символом * обозначается операчия свертки в пространстве $\mathscr{E}\left(\mathbb{C} \times S^{2 n-1}\right)$ по первой переменной.

ДокАЗАТЕЛЬСТво ЛЕмМЫ 1 . Покажем, что для любой функции $\varphi \in \mathscr{D}\left(\mathbb{C}^{n}\right)$ верно

$$
\int_{\mathbb{C}^{n}}\left\{\left(\alpha_{\varepsilon} * R^{*}[\psi]\right)(z)-R^{*}\left[\hat{\alpha}_{\varepsilon} *_{s} \psi\right](z)\right\} \varphi(z) d \omega_{2 n}(z)=0
$$

Из этого равенства будет следовать [10]. Имеем

$$
\int_{\mathbb{C}^{n}}\left(\alpha_{\varepsilon} * R^{*}[\psi]\right)(z) \varphi(z) d \omega_{2 n}(z)=\int_{\mathbb{C}^{n}}\left[R^{*} \psi\right](z)\left(\alpha_{\varepsilon} * \varphi\right)(z) d \omega_{2 n}(z) .
$$

Последний интеграл обозначим через $J$. Из (3) следует

$$
J=\int_{\mathbb{C} \times S^{2 n-1}} \psi(s, w) \widehat{\alpha_{\varepsilon} * \varphi}(s, w) d \omega_{2}(s) d \sigma(w),
$$

где $\widehat{\alpha_{\varepsilon} * \varphi}$ - преобразование Радона свертки $\alpha_{\varepsilon} * \varphi$. В силу формулы преобразования Радона свертки [1, с. 163] верно

$$
\widehat{\alpha_{\varepsilon} * \varphi}(s, w)=\left(\hat{\alpha}_{\varepsilon} *_{s} \hat{\varphi}\right)(s, w) .
$$

Тогда

$$
J=\int_{\mathbb{C} \times S^{2 n-1}} \psi(s, w)\left(\hat{\alpha}_{\varepsilon} *_{s} \hat{\varphi}\right)(s, w) d \omega_{2}(s) d \sigma(w) .
$$

Последний интеграл равен

$$
\int_{\mathbb{C} \times S^{2 n-1}}\left(\psi *_{s} \hat{\alpha}_{\varepsilon}\right)(s, w) \hat{\varphi}(s, w) d \omega_{2}(s) d \sigma(w) .
$$

Тогда, вновь применяя (3), получаем

$$
J=\int_{\mathbb{C}^{n}} R^{*}\left[\hat{\alpha}_{\varepsilon} *_{s} \psi\right](z) \varphi(z) d \omega_{2 n}(z) .
$$

Таким образом, доказана формула (11), из которой следует (10). Лемма доказана.

Продолжим доказательство теоремы 1 . Пусть $K_{\varepsilon}$ - замкнутое $\varepsilon$-вздутие компакта $K$. Пусть $\widehat{F}_{\varepsilon}(s, w)$ - преобразование Радона функции $F_{\varepsilon}(z)$. Покажем, что supp $\widehat{F}_{\varepsilon} \subset \widehat{K}_{\varepsilon}$. Гиперплоскость $z:\langle z, w\rangle=s$ пересекает $K_{\varepsilon}$ тогда и только тогда, когда для некоторых $z^{1} \in K$ и $z^{2} \in \bar{B}^{n}(0, \varepsilon)$ верно $\left\langle z^{1}, w\right\rangle=s-\left\langle z^{2}, w\right\rangle$. Поэтому верно

$$
\widehat{K}_{\varepsilon}=\left\{\left(s+\bar{B}^{1}(0, \varepsilon), w\right) \mid(s, w) \in \widehat{K}\right\} .
$$


Пусть $h(s, w) \in \mathscr{D}\left(\mathbb{C} \times S^{2 n-1}\right), \operatorname{supp} h \cap \widehat{K}_{\varepsilon}=\varnothing$. Согласно $(7)$

$$
\left\langle R F_{\varepsilon}, h\right\rangle=\left\langle F_{\varepsilon}, R^{*}(h)\right\rangle=\left\langle F * \alpha_{\varepsilon}, R^{*}(h)\right\rangle=\left\langle F, \alpha_{\varepsilon} * R^{*}(h)\right\rangle .
$$

По лемме $1\left\langle F, \alpha_{\varepsilon} * R^{*}(h)\right\rangle=\left\langle F, R^{*}\left(\hat{\alpha}_{\varepsilon} *_{s} h\right)\right\rangle$. Тогда

$$
\left\langle R F_{\varepsilon}, h\right\rangle=\left\langle F, R^{*}\left(\hat{\alpha}_{\varepsilon} *_{s} h\right)\right\rangle=\left\langle R F, \hat{\alpha}_{\varepsilon} *_{s} h\right\rangle .
$$

Покажем, что $\widehat{K} \cap \operatorname{supp}\left(\hat{\alpha}_{\varepsilon} *_{s} h\right)=\varnothing$. Если $\left(s_{0}, w_{0}\right) \in \widehat{K} \cap \operatorname{supp}\left(\hat{\alpha}_{\varepsilon} *_{s} h\right)$, то, так как $\hat{\alpha}_{\varepsilon}(s, w)=0$ для $|s| \geqslant \varepsilon$, для некоторого $s_{1} \in \bar{B}^{1}(0, \varepsilon)$ имеем $\left(s_{0}+s_{1}, w_{0}\right) \in \operatorname{supp} h$. Но в силу $(12)\left(s_{0}+s_{1}, w_{0}\right) \in \widehat{K}_{\varepsilon}$, что противоречит условию $\operatorname{supp} h \cap \widehat{K}_{\varepsilon}=\varnothing$. Таким образом, $\widehat{K} \cap \operatorname{supp}\left(\hat{\alpha}_{\varepsilon} *_{s} h\right)=\varnothing$ и, так как $\operatorname{supp} R F \subset \widehat{K}$, из (13) следует $\left\langle R F_{\varepsilon}, h\right\rangle=0$. Следовательно,

$$
\operatorname{supp} \widehat{F}_{\varepsilon} \subset \widehat{K}_{\varepsilon} \text {. }
$$

Для завершения доказательства теоремы 1 необходима

Лемма 2. В условиях и обозначениях теоремы 1 для каждой точки $z_{0} \notin K$ существуют окрестность $V_{z_{0}} u \delta>0$ такие, что для $z \in V_{z_{0}} u \varepsilon \leqslant \delta$ верно $F_{\varepsilon}(z)=0$.

ДОкАЗАТЕЛЬСТво ЛЕммы 2. Пусть $z_{0} \notin K$. Тогда по условию существует точка $w_{0} \in S^{2 n-1}$ такая, что

$$
\left\{\lambda:\left\langle\lambda, w_{0}\right\rangle=\left\langle z_{0}, w_{0}\right\rangle\right\} \cap K=\varnothing
$$

и множество $\left\{\left\langle z, w_{0}\right\rangle\right\}_{z \in K}$ имеет связное дополнение. Тогда $\left(\left\langle z_{0}, w_{0}\right\rangle, w_{0}\right) \notin \widehat{K}$. Пусть

$$
A=\left\{s \in \mathbb{C} \mid\left(s, w_{0}\right) \in \widehat{K}\right\}, \quad A_{\varepsilon}=\left\{s \in \mathbb{C} \mid\left(s, w_{0}\right) \in \widehat{K}_{\varepsilon}\right\} .
$$

Из (12) следует, что $A_{\varepsilon}=A+\bar{B}^{1}(0, \varepsilon)$. Так как $\widehat{K}$ - компакт, то $A$ и $A_{\varepsilon}-$ компакты. Считая $\varepsilon \leqslant 1$, имеем $A \cup A_{\varepsilon} \subset B^{1}(0, R)$. По условию $\left\langle z_{0}, w_{0}\right\rangle \notin A$; тогда для некоторого $\gamma>0$ и любого $\lambda \in \bar{B}^{n}(0, \gamma)$ имеем $\left\langle z_{0}+\lambda, w_{0}\right\rangle \notin A$. Следовательно, вьпуклый компакт $\Gamma_{1}=\left\{\left\langle z_{0}+\bar{B}^{n}(0, \gamma), w_{0}\right\rangle\right\}$ не пересекается с $A$. Зафиксируем $s_{1} \in \mathbb{C},\left|s_{1}\right|>R$. Тогда $s_{1} \in \mathbb{C} \backslash A$. Так как $\mathbb{C} \backslash A$ связно, существует ломаная $\Gamma_{2}$, соединяющая точки $s_{1}$ и $\left\langle z_{0}, w_{0}\right\rangle$ и не пересекающаяся с $A$. Таким образом, $\left(\Gamma_{1} \cup \Gamma_{2}\right) \cap A=\varnothing$. Так как $\left(\Gamma_{1} \cup \Gamma_{2}\right)$ и $A$ - компакты, для некоторого $0<\delta<1$ и всех $\varepsilon \leqslant \delta$ имеем

$$
\left\{\left(\Gamma_{1} \cup \Gamma_{2}\right)+B^{1}(0, \delta)\right\} \cap\left\{A+\bar{B}^{1}(0, \varepsilon)\right\}=\varnothing,
$$

т.е. $\left\{\left(\Gamma_{1} \cup \Gamma_{2}\right)+B^{1}(0, \delta)\right\} \cap A_{\varepsilon}=\varnothing$. Так как $A_{\varepsilon} \subset B^{1}(0, R)$, полагая

$$
D=\{s \in \mathbb{C}:|s|>R\} \cup\left\{\left(\Gamma_{1} \cup \Gamma_{2}\right)+B^{1}(0, \delta)\right\},
$$

имеем $D \cap A_{\varepsilon}=\varnothing$. При этом $D$ - неограниченное, связное, открытое множество, содержащее любую точку $\left\langle z_{0}+\lambda, w_{0}\right\rangle, \lambda \in \bar{B}^{n}(0, \gamma)$. По определению $A_{\varepsilon}$ имеем $\left(D \times\left\{w_{0}\right\}\right) \cap$ $\widehat{K}_{\varepsilon}=\varnothing, \varepsilon \leqslant \delta$. Тогда из $(14)$ следует $\left(D \times\left\{w_{0}\right\}\right) \cap \operatorname{supp} \widehat{F}_{\varepsilon}=\varnothing$ для $\varepsilon \leqslant \delta$. По условию исходный функционал $F$ имеет компактньй носитель, поэтому носители функций $F_{\varepsilon}$ также компактны. Тогда по теореме 2 для $\varepsilon \leqslant \delta$ и любого $\lambda \in \bar{B}^{n}(0, \gamma)$ функции $F_{\varepsilon}$ обращаются в нуль на гиперплоскости $\left\{z:\left\langle z, w_{0}\right\rangle=\left\langle z_{0}+\lambda, w_{0}\right\rangle\right\}$. Следовательно, для $\varepsilon \leqslant \delta$ имеем $F_{\varepsilon}(z)=0, z \in \bar{B}^{n}\left(z_{0}, \gamma\right)$. Лемма доказана. 
Пусть $\varphi \in \mathscr{D}\left(\mathbb{C}^{n}\right)$ и носитель $\varphi$ не пересекается с $K$. Имеем

$$
\lim _{\varepsilon \rightarrow 0}\left\langle F_{\varepsilon}, \varphi\right\rangle=\langle F, \varphi\rangle
$$

Для каждой точки $z \in \operatorname{supp} \varphi$ по лемме 2 существуют $\delta(z)>0$ и шар $B^{n}(z, \gamma(z))$, в котором $F_{\varepsilon}(z)=0$ для $\varepsilon \leqslant \delta(z)$. Так как носитель функции $\varphi-$ компакт, то он может быть покрыт конечньп набором таких шаров $B^{n}\left(z_{k}, \gamma\left(z_{k}\right)\right)$. При этом во всех этих шарах $F_{\varepsilon}(z)=0$ для $\varepsilon \leqslant \delta_{0}$, где $\delta_{0}=\min \delta\left(z_{k}\right)$. Следовательно, для $\varepsilon \leqslant \delta_{0}$ функции $F_{\varepsilon}$ равны нулю на носителе $\varphi$. Тогда из (15) следует $\langle F, \varphi\rangle=0$. Так как здесь $\varphi-$ любая гладкая функция, носитель которой не пересекается с $K$, то supp $F \subset K$. Теорема доказана.

\section{СПИСОК ЦИТИРОВАННОЙ ЛИТЕРАТУРЫ}

[1] Гельфанд И. М., Граев М. И., Виленкин Н. Я. Интегральная геометрия и связанные с ней вопросы теории представлений. М.: Наука, 1962.

[2] Хелгасон С. Преобразование Радона. М.: Мир, 1983.

[3] Ludwig D. The Radon transform on Euclidean space // Comm. Pure Appl. Math. 1966. V. 19. P. 49-81.

[4] Sekerin A. B. A support theorem for the complex Radon transform // Collectanea Mathematica. 1999. V. 50. P. 221-227.

[5] Секерин А. Б. Применения преобразования Радона в теории аппроксимации. Уфа: Башкирск. научн. центр УрО АН СССР, 1991.

[6] Hertle A. Continuity of the Radon transform and its inverse on Euclidean spaces // Math. Zeitschr. 1983. V. 184. P. 165-192.

[7] Секерин А. Б. Применение преобразования Радона к аппроксимационным задачам многомерного комплексного анализа. Дисс. ... д.ф..-м.н. Уфа: Ин-т математики УрО РАН, 1992.

[8] Sekerin A. B. Representation of functions by logarithmic potentials and reducibility of analytic functions of several variables // Collectanea Mathematica. 1996. V. 47. P. 187-206.

[9] Хермандер Л. Теория распределений и анализ Фурье. М.: Мир, 1986. 